\title{
Prevalence of Refractive Errors in the Total Population and the Analysis of Myopic Progression in Adults Aged 20 to 39 in the Urban Area of Tirana, Albania
}

\author{
Jazxhi Kleves \\ Scientific Research Office, "LUARASI” University, Tirana, Albania \\ Email: klevesgenova@gmail.com
}

How to cite this paper: Kleves, J. (2021) Prevalence of Refractive Errors in the Total Population and the Analysis of Myopic Progression in Adults Aged 20 to 39 in the Urban Area of Tirana, Albania. Open Journal of Ophthalmology, 11, 293-312. https://doi.org/10.4236/ojoph.2021.114024

Received: October 6, 2021

Accepted: November 14, 2021

Published: November 17, 2021

Copyright (อ 2021 by author(s) and Scientific Research Publishing Inc. This work is licensed under the Creative Commons Attribution International License (CC BY 4.0).

http://creativecommons.org/licenses/by/4.0/ (c) (i) Open Access

\begin{abstract}
This study is a retrospective analysis of data stored digitally for 13 years in a Vision Center located in the urban area of Tirana, Albania. In a population of 19,686 people $(39.5 \% \mathrm{M}$ and $60.5 \% \mathrm{~F})$ who met the criteria, there was a prevalence of astigmatism $42 \%$ (12\% only astigmatism, $30 \%$ associated with hyperopia or myopia), myopia $25 \%$, hyperopia $22 \%$ and $24 \%$ presbyopic prescriptions for the medium and near distance. The myopia progression study (mean spherical equivalent) indicates that $20 \%$ of people had no change, $42 \%$ had an increase in myopic correction from $0.25 \mathrm{D}$ to $0.50 \mathrm{D}, 17 \%$ from $0.75 \mathrm{D}$ to $1.00 \mathrm{D}, 11 \%$ from $1.25 \mathrm{D}$ to $1.50 \mathrm{D}, 5 \%$ from $1.75 \mathrm{D}$ to $2.00 \mathrm{D}$ and $5 \%$ of the population had myopic progression greater than $2.00 \mathrm{D}$.
\end{abstract}

\section{Keywords}

Myopia, Hyperopia, Astigmatism, Myopic Progression, Refractive Error,

Presbyopia, Albania

\section{Introduction}

Refractive errors are the most common eye problems globally and the second leading cause of treatable blindness, which affects quality of life, school and work performance. Worldwide, $21 \%$ of adults reported visual impairment and the rate varies by country's income status resulting $24 \%$ in low, $23 \%$ in middle, and $13 \%$ in high-income countries [1]. The prevalence of refractive errors has been reported in many international studies [2] [3] [4]. Statistics on the distribution of the error among the population using optical correction also offer important in- 
formation. Analysis help Healthcare Providers improve management, implement containment plans and prevention strategies.

A large part of this commitment concerns myopic progression. Genetic and ethnic risk factors have been studied on a large scale recently and are closely related to environmental, socio-cultural and lifestyle factors in several international studies [5]-[11].

\section{Objectives}

The objectives of this study are to describe the general prevalence of refractive errors and to analyze the progression of myopia from $-1.00 \mathrm{D}$ to $-4.75 \mathrm{D}$ (mean spherical equivalent) in adults aged 20 to 39 by studying a 13-year database of the ordered glasses and refractive error prescriptions in the urban area of Tirana, Albania.

\section{Materials and Methods}

\subsection{Participants, Inclusion and Exclusion Criteria}

This study analyzed the prevalence of refractive errors in the entire population of people who ordered glasses or had eye exams from 01, January 2008 to 01, October 2020 in the Vision Center located in the city of Tirana. Myopic progression (mean spherical equivalent) was then analyzed in the population aged 20 to 39 years in 2020, which recorded at least one prescription for myopia from $-1.00 \mathrm{D}$ to $-4.75 \mathrm{D}$ from 01, January 2016 to 01 , October 2020. For the people included, the previous prescriptions were also taken into consideration but at least one visit was required starting from 2016, a period in which the co-management protocol was applied in the Vision Center. The examinations carried out in the Health Prevention Room together with the reports issued by other clinics or hospitals made it possible to strictly comply with the exclusion criteria.

Since 2008 the Vision Center offers products in the Optics Shop and eye exams in a separate area to which the Medical Prevention Room was added later in 2016. This study analyzed the commercial database of the Optical Shop of ordered glasses, quotes to buy glasses, consultations for the choice of ophthalmic lenses including information on refractive error and its optical correction explained to the customer. Each file recorded data on previous glasses made in other stores, including prescription, lens type, how the glasses were used, perceived problems and what the expectations were. Persons who had undergone refractive surgery or people whose optical correction of refractive error could be altered by problems regarding the retina, opacity of the lens, irregularities of the surface, lesion to the cornea, complications of ocular health or general pathologies, were excluded from the current study.

\subsection{Population}

The study of the prevalence of refractive errors involved 19,686 people who met the criteria and their age was represented in 5-years groups. The first two groups 
were less numerous: 5 - 9 years old group (4\%) and 10 - 14 years old group (6\%) because eye care is mainly offered by hospitals and pediatric clinics. The age distribution for adults was similar to the demographic data of the resident population in Tirana. In brackets are marked the 2020 data from the Municipality of Tirana [12]. It appears that $45 \%(46.07 \%)$ of the population in this study was under 35 years, $77 \%$ (70.30\%) in working age (15 - 64 years old), 27\% (24.58\%) of residents over 55 years old, and $13 \%(12.70 \%)$ of the elderly population was over 65 years old.

Regarding Sex, in the total population of 19,686 people, $39.5 \%$ were male and $60.5 \%$ female and a similar proportion was also found in studies performed on a population that already has an optical correction in use [13]. Males outnumbered females under the age of 20 and slightly in the 45 - 49 years old group; females prevailed in the rest of the population. In more numerous age groups, the prevalence of women was much higher: 486 males and 796 females (20 - 24 years old group), 577 males and 1219 females (25 - 29 years old group), 742 males 1376 females (30 - 34 years old group), 526 males and 999 females (35 - 39 years old group), 551 males and 813 females (55 - 59 years old group), 413 males and 694 females (60 - 64 years old group).

New people registered every year. The number of people registered for the first time was 703 in 2008 and gradually grew to 1947 people in 2013, 2145 in 2015, and 2144 new people in 2016. There were several exams done each year by people who have returned for a second time (or more), which was $4 \%$ of the total number of visits in 2011, 14\% in 2012, 26\% in 2013,34\% in 2014 and $36 \%$ in 2015. From 2016 onwards, approximately $50 \%$ of the exams each year were taken by people who returned for a second, third, or more exams in the Vision Center.

\subsection{Material and Instruments}

The data of the eyeglasses ordered and the prescription of the refractive errors were recorded from 01 January 2016 to 01 October 2020 with a specific software for the Vision Center: WinOttica licensed by SBM Sistemi S.r.l., Turin-Italy.

The file of a person contained in the first section the personal data, the time and the number of all services provided. The technical section showed the prescription sheets for refractive errors. For each exam or ordered glasses, a new sheet was created and automatically added to the previous ones. There were separate spaces for the sphere, cylinder and axis followed by space for the prism and base. There was a line for distance glasses, a second line for the middle zone and a third line for near use. All dioptric values were entered with $0.25 \mathrm{D}$ intervals and the astigmatism axis with 5-degree intervals.

The system allowed the optician and optometrist to change the sign of the cylinder and automatically shift the axis by 90 degrees. However, the prescriptions were registered in the database in the way that optical laboratories that assemble the glasses preferred. The TABO system was used for astigmatism: both the left 
and right eye axis were measured counterclockwise. The component of the sphere and the cylinder were marked with the same sign, so we found a correction with a positive sphere combined with a positive cylinder and a negative sphere correction combined with a negative cylinder. Where the value of the cylinder was higher than the value of the sphere and did not have the same sign, we considered mixed astigmatism with opposite signs. If no myopia or hyperopia were present, the sphere was marked with $0.00 \mathrm{D}$ and the correction was considered as a cylinder only, marked with a positive or negative sign.

Optometrists were able to perform simple or multiple searches by combining age and gender with the prescription data. The software extracted the list of people who had in common the search criteria at least in one eye. If the refractive error was composed of a sphere, cylinder, near addition or had different dioptric power between one eye and the other, the same person could be part of different groups at the same time according to the search criteria. There were other detailed datasheets for optometric examination and contact lenses but they were not included in this study.

In the Descriptive Notes section, information or problems relating to glasses previously ordered in other centers, prescriptions issued by other clinics, particular visual or work needs, family history, etc. have been noted. This information could only be obtained by manually accessing each individual folder and so we did in the study on myopic progression.

The data extracted from the WinOttica archive by SBM Sistemi S.r.l. were later inserted into Microsoft Office Excel Worksheet to make tables and calculate statistics in numerical order and in percentage terms.

Study of the prevalence of refractive error. The dioptric power was managed in five groups: from $0.25 \mathrm{D}$ to $0.75 \mathrm{D}$, from $1.00 \mathrm{D}$ to $1.75 \mathrm{D}$, from $2.00 \mathrm{D}$ to $2.75 \mathrm{D}$, from $3.00 \mathrm{D}$ to $3.75 \mathrm{D}$, from $4.00 \mathrm{D}$ to $4.75 \mathrm{D}$. The prevalence and distribution in age groups were analyzed in detail for the refractive errors with spherical power from $-4.75 \mathrm{D}$ to $+4.75 \mathrm{D}$ and cylindrical power from $-4.00 \mathrm{D}$ to $+4.00 \mathrm{D}$. The number of people with refractive errors higher than these values was low and had a limited weight on the entire population.

Study of the myopic progression. The myopic progression study involved adults aged 20 - 39 in 2020 that had recorded at least one prescription from $-1.00 \mathrm{D}$ to $-4.75 \mathrm{D}$ since 2016 when the co-management protocol was applied in the Vision Center. The examinations carried out in the health prevention room and the medical reports issued by other clinics or hospitals allowed to strictly comply with the exclusion criteria. Minimal corrections up to $-0.75 \mathrm{D}$, presented in 1291 files, of which 836 files belonging to people between the age of 20 and 39, were excluded from the study of myopic progression. Corrections of myopia higher than $-4.75 \mathrm{D}$, present in 185 files (110 between age 20 and 39) were also excluded.

All the prescriptions since 2008, belonging to people who meet the inclusion criteria, have been analyzed. Myopia was managed in four groups: from $-1.00 \mathrm{D}$ 
to $-1.75 \mathrm{D}$, from $-2.00 \mathrm{D}$ to $-2.75 \mathrm{D}$, from $-3.00 \mathrm{D}$ to $-3.75 \mathrm{D}$ and from $-4.00 \mathrm{D}$ to $-4.75 \mathrm{D}$. In prescriptions where myopia is associated with astigmatism, the spherical mean equivalent was used to assess myopic progression. Dioptric increase was reported in $0.50 \mathrm{D}$ step change groups: $0.00 \mathrm{D}$ (no change), change $0.25 \mathrm{D}$ $0.50 \mathrm{D}$, change $0.75 \mathrm{D}-1.00 \mathrm{D}$, change $1.25 \mathrm{D}-1.50 \mathrm{D}$, change $1.75 \mathrm{D}-2.00 \mathrm{D}$, etc.

\section{Results}

After the exclusion of subjects with ocular pathology, the population was made up of 19,686 people $(39.5 \% \mathrm{M}$ and $60.5 \% \mathrm{~F})$ who were examined from 01 , January 2008 to 01 , October 2020.

Hyperopia. We noted that 4259 people (38.9\% M and $61.1 \% \mathrm{~F}$ ) had hyperopic prescriptions. There were 2048 people aged from 5 to 79 years with a spherical refractive error in at least one eye from $+0.25 \mathrm{D}$ to $+0.75 \mathrm{D}, 1902$ people with an error from $+1.00 \mathrm{D}$ to $+1.75 \mathrm{D}, 821$ people from $+2.00 \mathrm{D}$ to $+2.75 \mathrm{D}, 356$ people from $+3.00 \mathrm{D}$ to $+3.75 \mathrm{D}, 168$ people from $+4.00 \mathrm{D}$ to $4.75 \mathrm{D}$ The general distribution across all age groups is shown in Table 1.

The total number of spherical corrections for far and near distances above $+5.00 \mathrm{D}$ was very limited. We found 52 people 5 - 19 years old, 30 people 20 - 34 years old, 19 people 35 - 49 years old, 44 people 50 - 64 years old and 77 people from 65 - 79 years old.

Table 1. Distribution of hyperopia in age groups.

\begin{tabular}{|c|c|c|c|c|c|}
\hline \multirow{2}{*}{ Age Groups } & \multicolumn{5}{|c|}{ People with Positive Spherical Lenses } \\
\hline & $+0.25 /+075$ & $+1.00 /+1.75$ & $+2.00 /+2.75$ & $+3.00 /+3.75$ & $+4.00 /+4.75$ \\
\hline $5 / 9$ years old & 53 & 59 & 33 & 29 & 20 \\
\hline 10/14 years old & 123 & 119 & 67 & 55 & 31 \\
\hline $15 / 19$ years old & 118 & 97 & 61 & 31 & 15 \\
\hline $20 / 24$ years old & 122 & 65 & 30 & 12 & 8 \\
\hline $25 / 29$ years old & 112 & 51 & 26 & 21 & 7 \\
\hline $30 / 34$ years old & 147 & 76 & 44 & 24 & 19 \\
\hline $35 / 39$ years old & 97 & 58 & 31 & 18 & 7 \\
\hline 40/44 years old & 94 & 65 & 37 & 20 & 10 \\
\hline $45 / 49$ years old & 114 & 73 & 30 & 14 & 1 \\
\hline $50 / 54$ years old & 161 & 158 & 45 & 18 & 5 \\
\hline $55 / 59$ years old & 268 & 268 & 86 & 26 & 6 \\
\hline $60 / 64$ years old & 258 & 288 & 110 & 30 & 12 \\
\hline $65 / 69$ years old & 218 & 281 & 103 & 26 & 14 \\
\hline 70/74 years old & 116 & 160 & 71 & 24 & 7 \\
\hline $75 / 79$ years old & 47 & 84 & 47 & 8 & 6 \\
\hline
\end{tabular}


Astigmatism. The sphere component and the cylinder were marked with the same sign in the database, resulting in correction of hyperopia combined with a positive cylinder and myopia combined with a negative cylinder. Where sphere was marked with $0.00 \mathrm{D}$ was considered as a cylinder alone positive (present in 943 people) or cylinder alone negative (present in 1439 people). Other 1179 people ( $46 \%$ male and $54 \%$ female) aged from 5 to 79 years had at least one eye presenting mixed astigmatism error, with cylindrical component higher and with an opposite sign from the sphere. In the age 5 - 19 year-old groups there were 242 people and in the 20 - 34 years old groups we found the highest number (369 people).

Positive astigmatism associated with hyperopia was present in 3372 people (42.7\% $\mathrm{M}$ and $57.3 \mathrm{~F}$ ) aged from 5 to 79 years. The population with positive cylindrical correction from +0.25D to +0.75D was 2369 and from +1.00D to +1.75D was 768 . There were 657 people from $+2.00 \mathrm{D}$ to $+2.75 \mathrm{D}$ and 106 people with correction from $+3.00 \mathrm{D}$ to $+4.00 \mathrm{D}$. The general distribution can be seen in $\mathrm{Ta}$ ble 2.

Negative astigmatism associated with myopia was present in 3750 people (42\% $\mathrm{M}$ and $58 \% \mathrm{~F}$ ). We found 2603 people with negative cylindrical correction from $-0.25 \mathrm{D}$ to $-0.75 \mathrm{D}, 1170$ people from $-1.00 \mathrm{D}$ to $-1.75 \mathrm{D}, 493$ people from $-2.00 \mathrm{D}$ to $-2.75 \mathrm{D}, 237$ people from $-3.00 \mathrm{D}$ to $-4.00 \mathrm{D}$. The general distribution across all age groups is shown in Table 3 .

Table 2. Distribution of positive astigmatism associated with hyperopia in age groups.

\begin{tabular}{ccccc}
\hline \multirow{2}{*}{ Age Groups } & \multicolumn{4}{c}{ People with Positive Cylindrical Lenses } \\
\cline { 2 - 5 } & $+\mathbf{+ 0 . 2 5 / + 0 . 7 5}$ & $\mathbf{+ 1 . 0 0 / + 1 . 7 5}$ & $\mathbf{+ 2 . 0 0 / + 2 . 7 5}$ & $\mathbf{+ 3 . 0 0 / + 4 0 0}$ \\
\hline 5/9 years old & 41 & 57 & 23 & 11 \\
10/14 years old & 102 & 90 & 47 & 19 \\
15/19 years old & 74 & 70 & 32 & 14 \\
20/24 years old & 55 & 33 & 20 & 8 \\
25/29 years old & 84 & 44 & 14 & 7 \\
30/34 years old & 102 & 59 & 24 & 18 \\
35/39 years old & 83 & 42 & 13 & 5 \\
40/44 years old & 89 & 23 & 9 & 7 \\
$45 / 49$ years old & 156 & 49 & 8 & 3 \\
$50 / 54$ years old & 235 & 75 & 49 & 5 \\
$55 / 59$ years old & 340 & 65 & 136 & 1 \\
$60 / 64$ years old & 362 & 54 & 136 & 1 \\
$65 / 69$ years old & 362 & 50 & 90 & 2 \\
$70 / 74$ years old & 195 & 42 & 39 & 3 \\
$75 / 79$ years old & 89 & 15 & 17 & 2 \\
\hline
\end{tabular}


Table 3. Distribution of negative astigmatism associated with Myopia in age groups.

\begin{tabular}{|c|c|c|c|c|}
\hline \multirow{2}{*}{ Age Groups } & \multicolumn{4}{|c|}{ People with Negative Cylindrical Lenses } \\
\hline & $-0.25 /-0.75$ & $-1.00 / 1.75$ & $-2.00 /-2.75$ & $-3.00 /-4.00$ \\
\hline $5 / 9$ years old & 31 & 44 & 27 & 9 \\
\hline $10 / 14$ years old & 92 & 76 & 39 & 17 \\
\hline $15 / 19$ years old & 206 & 95 & 51 & 33 \\
\hline $20 / 24$ years old & 334 & 123 & 53 & 19 \\
\hline $25 / 29$ years old & 438 & 162 & 78 & 45 \\
\hline $30 / 34$ years old & 495 & 197 & 85 & 33 \\
\hline $35 / 39$ years old & 369 & 133 & 54 & 26 \\
\hline $40 / 44$ years old & 206 & 81 & 27 & 18 \\
\hline $45 / 49$ years old & 102 & 70 & 23 & 11 \\
\hline $50 / 54$ years old & 96 & 48 & 17 & 7 \\
\hline $55 / 59$ years old & 76 & 41 & 11 & 6 \\
\hline $60 / 64$ years old & 61 & 23 & 3 & 7 \\
\hline $65 / 69$ years old & 48 & 31 & 11 & 2 \\
\hline $70 / 74$ years old & 36 & 32 & 6 & 2 \\
\hline $75 / 79$ years old & 13 & 14 & 8 & 2 \\
\hline
\end{tabular}

Positive lens correction for the intermediate and near distance. The population with a positive lens correction for the intermediate and near distance counted 4765 people $(39.7 \% \mathrm{M}$ and $60.3 \% \mathrm{~F})$. In this population aged from 5 to 79 years, 806 people at least in one eye had a correction from $+0.25 \mathrm{D}$ to $+0.75 \mathrm{D}$, 1478 spherical error from $+1.00 \mathrm{D}$ to $+1.75 \mathrm{D}, 1585$ people from $+2.00 \mathrm{D}$ to $+2.75 \mathrm{D}$, 851 people from $+3.00 \mathrm{D}$ to $+3.75 \mathrm{D}, 325$ people from $+4.00 \mathrm{D}$ to $+4.75 \mathrm{D}$. The general distribution is shown in Table 4.

Myopia was present in 4863 people (34.6\% M and $65.4 \% \mathrm{~F}$ ). In this population 2464 people, at least in one eye had an error from $-0.25 \mathrm{D}$ to $-075 \mathrm{D}, 1920$ people from $-1.00 \mathrm{D}$ to $-1.75 \mathrm{D}, 1121$ people from $-2.00 \mathrm{D}$ to $-2.75 \mathrm{D}$, 664 people from $-3.75 \mathrm{D}$ to $-3.75 \mathrm{D}$, 397 people from $-4.00 \mathrm{D}$ to $-4.75 \mathrm{D}$. Only 318 people had a higher myopic correction.

Analyzing the prevalence of people who had registered for the first time each year, we noted that in 2008 and 2009 the group of people with hyperopia exceeded that of myopia. Subsequently, this ratio was reversed and since 2010 the population with myopia correction was more numerous especially in the lower powers groups with a spherical correction from $-0.25 \mathrm{D}$ to $-2.75 \mathrm{D}$. The general distribution of myopia in age groups is shown in Table 5.

The distribution of refractive errors was analyzed in 5 power groups for the sphere and 4 for the cylinder. The data provided in the tables includes people with anisometropia who belong to two groups. They were counted twice: 1036 
Table 4. Distribution of positive sphere correction for medium and near distance in age groups.

\begin{tabular}{cccccc}
\hline & \multicolumn{5}{c}{ People with Positive Spherical Lenses } \\
\cline { 2 - 6 } Age Groups & $+\mathbf{0 . 2 5 / + 0 . 7 5}$ & $+\mathbf{1 . 0 0 / + 1 . 7 5}$ & $\mathbf{+ 2 . 0 0 / + 2 . 7 5}$ & $\mathbf{+ 3 . 0 0 / 3 . 7 5}$ & $\mathbf{+ 4 . 0 0 / + 4 . 7 5}$ \\
\hline $30 / 34$ years old & 57 & 10 & 0 & 0 & 0 \\
$35 / 39$ years old & 70 & 16 & 0 & 0 & 0 \\
40/44 years old & 111 & 29 & 0 & 0 & 0 \\
$45 / 49$ years old & 215 & 229 & 57 & 10 & 1 \\
$50 / 54$ years old & 223 & 479 & 192 & 43 & 12 \\
$55 / 59$ years old & 97 & 443 & 461 & 147 & 43 \\
$60 / 64$ years old & 19 & 178 & 437 & 216 & 69 \\
$65 / 69$ years old & 6 & 55 & 269 & 230 & 82 \\
$70 / 74$ years old & 8 & 23 & 125 & 142 & 75 \\
$75 / 79$ years old & 0 & 16 & 44 & 63 & 43 \\
\hline
\end{tabular}

Table 5. Distribution of Myopia in age groups.

\begin{tabular}{|c|c|c|c|c|c|}
\hline \multirow{2}{*}{ Age Groups } & \multicolumn{5}{|c|}{ People with Negative Spherical Lenses } \\
\hline & $-0.25 /-075$ & $-1.00 /-1.75$ & $-200 /-275$ & $-300 /-3.75$ & $-4.00 /-4.75$ \\
\hline $5 / 9$ years old & 19 & 19 & 11 & 1 & 2 \\
\hline $10 / 14$ years old & 82 & 109 & 71 & 40 & 21 \\
\hline $15 / 19$ years old & 214 & 243 & 162 & 104 & 54 \\
\hline $20 / 24$ years old & 307 & 305 & 213 & 141 & 70 \\
\hline $25 / 29$ years old & 474 & 355 & 186 & 94 & 61 \\
\hline $30 / 34$ years old & 535 & 339 & 197 & 113 & 63 \\
\hline $35 / 39$ years old & 338 & 220 & 93 & 57 & 56 \\
\hline $40 / 44$ years old & 180 & 95 & 63 & 31 & 21 \\
\hline $45 / 49$ years old & 81 & 60 & 41 & 19 & 12 \\
\hline $50 / 54$ years old & 78 & 54 & 26 & 17 & 15 \\
\hline $55 / 59$ years old & 64 & 39 & 18 & 15 & 7 \\
\hline $60 / 64$ years old & 34 & 26 & 14 & 9 & 4 \\
\hline $65 / 69$ years old & 28 & 24 & 12 & 11 & 7 \\
\hline $70 / 74$ years old & 21 & 17 & 12 & 7 & 3 \\
\hline $75 / 79$ years old & 9 & 15 & 2 & 5 & 1 \\
\hline
\end{tabular}

people with hyperopia, 1703 with myopia, 528 with positive cylinder, 753 with negative cylinder, and 280 people with positive spherical correction for near and medium range. 
Myopic progression in adults (20 - 39 years old) This study focused on people between the ages of 20 and 39, who registered in the database at least one prescription for the correction of the refractive error of myopia from $-1.00 \mathrm{D}$ to $-4.75 \mathrm{D}$ in the period from 01.01.2016 to 01.10.2020. To assess myopic progression, every single prescription that those people had previously registered in the Vision Center (as of January 1, 2008) was verified and included in the study. In cases of myopia associated with astigmatism, we used the spherical equivalent to evaluate myopic progression. We did not include in the study minimal corrections from $-0.25 \mathrm{D}$ to $-0.75 \mathrm{D}$ that were present in 1291 files, of which 836 files were people aged between 20 and 39 years. We also did not include corrections of myopia equal to or greater than $-5.00 \mathrm{D}$ that were present in 185 files (110 between 20 and 39 years).

The power of refractive error from $-1.00 \mathrm{D}$ to $-4.75 \mathrm{D}$ was organized into four groups and manually each of the 1464 files (29\% men and $71 \%$ women) were checked. A total of $25 \%$ people were in two groups because they have different refractive errors between their two eyes. After identifying the duplicates it turned out that the 1464 files belonged to 1091 people. Refractive errors with spherical power from $-1.00 \mathrm{D}$ to $-1.75 \mathrm{D}$ resulted in 689 files ( 516 people), from $-2.00 \mathrm{D}$ to $-2.75 \mathrm{D}$ in 387 files (272 people). From $-3.00 \mathrm{D}$ to $-3.75 \mathrm{D}$ the number decreased in 243 files ( 166 people), and from $-4.00 \mathrm{D}$ to $-4.75 \mathrm{D}$ in 145 files (137 people).

A sum of 365 people, who only took one eye exam in these 5 years, were not included in this part of the study which is based on the differences in myopic correction between two exams done with the same co-management protocol in this Vision Center. A total of 726 people and 2751 refractive error tests were included. By calculating the number of exams per capita, 244 people recorded 2 exams, 160 people 3 exams, 116 people 4 exams, 77 people 5 exams, 42 people 6 exams, 25 people 7 exams, 29 people 8 exams and other 29 people 9 - 11 exams.

The difference in dioptric power in the prescriptions was examined and in the presence of astigmatism, the spherical equivalent was considered equal to the power of the sphere plus half of the cylindrical power. It turned out that 149 people had no prescription change, 306 people had an increase from $0.25 \mathrm{D}$ to $0.50 \mathrm{D}, 126$ people an increase from $0.75 \mathrm{D}$ to $1.00 \mathrm{D}, 79$ people from $1.25 \mathrm{D}$ to $1.50 \mathrm{D}, 41$ people from $1.75 \mathrm{D}$ to $2.00 \mathrm{D}, 18$ people from $2.25 \mathrm{D}$ to $2.50 \mathrm{D}$ and 15 people had an increase greater than $2.50 \mathrm{D}$. There were 8 people with different myopic progression between the two eyes and resulted in two groups at the same time. There were 833 exams where the prescription had not been changed, where 307 belonged to the power group from $-1.00 \mathrm{D}$ to $-1.75 \mathrm{D}, 218$ from $-2.00 \mathrm{D}$ to $-2.75 \mathrm{D}, 158$ from $-3.00 \mathrm{D}$ to $-3.75 \mathrm{D}$ and 150 from $-4.00 \mathrm{D}$ to $-4.75 \mathrm{D}$.

Analyzing the myopic progression within the 4 dioptric power groups, it appeared that in the group with a myopic correction from $-1.00 \mathrm{D}$ to $-1.75 \mathrm{D}, 25 \%$ of people had no change in refractive error, $53 \%$ had an increase in myopia from $0.25 \mathrm{D}$ to $0.50 \mathrm{D}, 18 \%$ from $0.75 \mathrm{D}$ to $1.00 \mathrm{D}$ and $4 \%$ from $1.25 \mathrm{D}$ to $1.50 \mathrm{D}$. In the group with myopic refractive error from $-2.00 \mathrm{D}$ to $-2.75 \mathrm{D}, 21 \%$ of people main- 
tained the same correction, $37 \%$ had an increase in myopia from $0.25 \mathrm{D}$ to $0.50 \mathrm{D}$, $19 \%$ an increase from $0.75 \mathrm{D}$ to $1.00 \mathrm{D}, 15 \%$ from $1.25 \mathrm{D}$ to $1.50 \mathrm{D}, 6 \%$ from 1.75 to $2.00 \mathrm{D}$ and $2 \%$ had an increase greater than $2.25 \mathrm{D}$. In the refractive error from $-3.00 \mathrm{D}$ to $-3.75 \mathrm{D}$ we noted that the number of people who had no changes in the correction decreased to $11 \%$, while $34 \%$ of people had an increase in myopia from $0.25 \mathrm{D}$ to $0.50 \mathrm{D}, 25 \%$ an increase from $0.75 \mathrm{D}$ to $1.00 \mathrm{D}, 18 \%$ from $1.25 \mathrm{D}$ to $1.50 \mathrm{D}, 9 \%$ from $1.75 \mathrm{D}$ to $2.00 \mathrm{D}$ and $8 \%$ had an increase greater than $2.25 \mathrm{D}$. People with the myopic refractive error between $-4.00 \mathrm{D}$ to $-4.75 \mathrm{D}$ represented a wider range of variation in myopic progression: $20 \%$ kept the optical correction unchanged, $30 \%$ had an increase in myopia from $0.25 \mathrm{D}$ to $0.50 \mathrm{D}, 13 \%$ from $0.75 \mathrm{D}$ to $1.00 \mathrm{D}, 12 \%$ from $1.25 \mathrm{D}$ to $1.50 \mathrm{D}, 12 \% 1.75 \mathrm{D}$ to $2.00 \mathrm{D}, 5 \% 2.25 \mathrm{D}$ to $2.50 \mathrm{D}, 2 \% 2.75 \mathrm{D}$ to $3.00 \mathrm{D}, 4 \% 3.25$ to $4.00 \mathrm{D}$ and $2 \%$ of people had an increase greater than $4.25 \mathrm{D}$. Figure 1 shows the change from $0.00 \mathrm{D}$ to $2.50 \mathrm{D}$ in the four power groups.

The time span in which the prescriptions were recorded was also evaluated. So, myopic progression occurred in 1 year for 52 people, 2 years for 162 people, 3 years for 97 people, 4 years for 91 people, 5 years for 89 people, 6 years for 73 people, 7 years for 61 people, 8 years for 31 people, 9 years for 44 people and 10 13 years for 26 people

\section{Discussion}

More than 40,000 prescriptions (belonging to 19,686 people) personally recorded by the author for a period of 13 years were analyzed. The results were compared with the statistics of international scientific publications. The prevalence

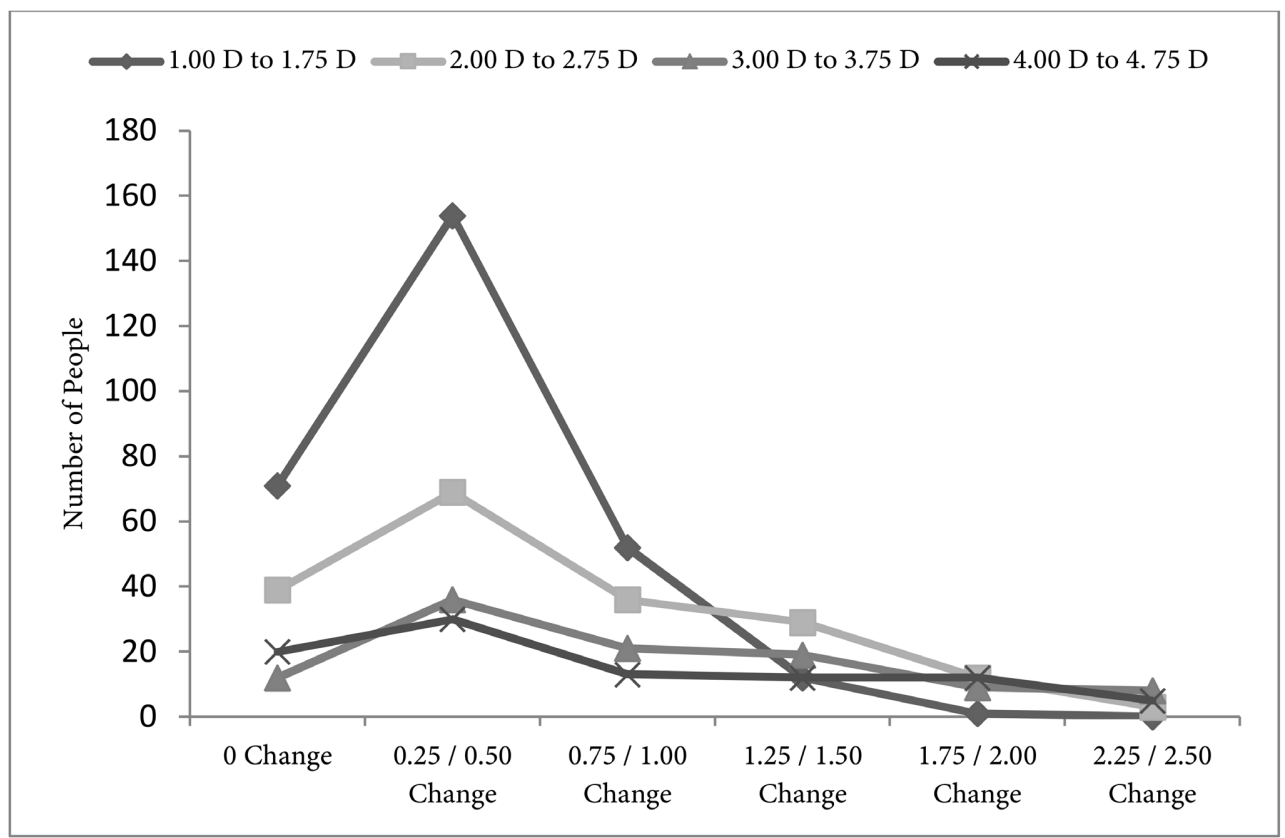

Figure 1. The change in refractive error of Myopia from $-1.00 \mathrm{D}$ to $-4.75 \mathrm{D}$ in four power groups. 
reported in this study is Astigmatism 42\% (12\% only astigmatism, 30\% associated with hyperopia or myopia), myopia $25 \%$, hyperopia $22 \%$ and appears similar with data from adults in Europe (respectively 39.7\%, 36\%, 23\%) according to a systematic global review of refractive errors [2]. The group of children aged 5 to 14 is less than $10 \%$ of the total population of this study, with a prevalence of astigmatism error 39\%, hyperopia 31\%, myopia 20\%. A more detailed analysis and comparison with international data was performed for each refractive error.

\subsection{Analysis of the Prevalence of Refractive Errors}

Hyperopia. As found in many studies, age affects the prevalence and distribution of hyperopia in the population. In the case of hyperopia from $+0.25 \mathrm{D}$ to $+1.75 \mathrm{D}$, there is an average presence of $5 \%-6 \%$ for each group up to age 49 and $8 \%$ in the 50 - 54 years old group. The prevalence rises from $13 \%$ to $15 \%$ in the $55-69$ years old groups and $6 \%-8 \%$ in the $70-74$ years old group. For powers from $+2.00 \mathrm{D}$ to $+2.75 \mathrm{D}$ the prevalence has peaks of $7 \%-8 \%$ in the groups of age 10 to 19 and $10 \%-13 \%$ for the age from 55 to 69 . Powers greater than $+3.00 \mathrm{D}$ are more present in the age between 5 and 19 with the peak in the age from 10 to 14. We note that the distribution of hyperopia (Table 1 ) and positive astigmatism (Table 2) in general follows the same trend.

The reviews that analyzed the studies report that different cut points are applied to establish the prevalence of hyperopia. The global mean prevalence in adults was 30.6\%, in Africa 38.6\%, in Americas 37.2\%, while Europe had the lowest presence: $23.1 \%$. From a study in the United States, the mean prevalence of hyperopia was $38.2 \%$ with the maximum level of $50.2 \%$ found in Hispanic participants [2] [14].

Uncorrected hyperopia leads to imbalances in the relationship between convergence and accommodation and in pediatric ages also to amblyopia in many cases. This was also found in this study.

Poor vision and amblyopia can affect learning, academic performance and in the future limit choice in profession and other daily activities. Anisometropia of $0.75 \mathrm{D}$ astigmatism or $1.5 \mathrm{D}$ sphere can lead to amblyopia which is deeper in cases anisohyperopia and anisoastigmatism. However, there have been improvements in recent years. Multimodal protocols which also include binocular exercises and particular optical corrections of glasses and contact lenses are more effective. It increases the probability of success and the critical age when amblyopia is treatable or reversible [15] [16] [17].

On the other hand, many glasses with low positive powers should not be prescribed to children according to international guidelines [18]-[23]. Headaches, fatigue and image distortion are often linked to poor frame fit and inadequate interpupillary distance of the lenses, especially in children [8].

Astigmatism: 2382 people, $12 \%$ of the entire population, have at least in one eye the refractive error of astigmatism, without spherical correction. More than $30 \%$ of the general population has the cylindrical component combined with 
myopia (3236 people) or hyperopia (3143 people). They have a cylinder from $-0.25 \mathrm{D}$ to $-0.75 \mathrm{D} 2670$ people, $76 \%$ of whom are also myopic while $92 \%$ of the 2455 people with astigmatism from $+0.25 \mathrm{D}$ to $+0.75 \mathrm{D}$, are also hyperopic. Astigmatism showed many variations in the different regions with an average value of $40.4 \%$. The highest prevalence was $45.6 \%$ in the Americas, $44.8 \%$ in Southeast Asia and the lowest in Africa 11.4\% [2].

The difficulties of accommodation in the presence of astigmatism depend on the extent of the error, whether it is undercorrected or totally uncorrected and in children it can also lead to amblyopia [15] [16] [17] [24] [25].

Prolonged use of electronic devices increases the risk of astigmatism in children while in many adult prescriptions in this study the cylindrical component is not confirmed by topography and keratometry [26]. Physiological astigmatism often is considered a refractive error and was included in many previous prescriptions where the auto-refractometer print is also attached. Despite attempts to reduce this phenomenon, 2451 people or $30 \%$ of the population with astigmatism in this study have a cylindrical correction of $0.25 \mathrm{D}$.

Positive lenses used in the near and medium distance. In the population of 5045 people with positive prescription in near and medium distance vision, $16 \%$ use a spherical power from $+0.25 \mathrm{D}$ to $+0.75 \mathrm{D}$ starting from age 30 with the highest prevalence from age 45 to $59 ; 29 \%$ of this population uses a correction from $+1.00 \mathrm{D}$ to $+1.75 \mathrm{D}$ with a minimum presence at $30-44$ years old group and the highest prevalence from age 50 to $59 ; 31 \%$ use spherical power from $+2.00 \mathrm{D}$ to $+2.75 \mathrm{D}$ starting from 45 - 49 years old group, with a higher concentration from age 55 to 64 and a significant presence even from age 65 to 74 . The two groups that use the spherical power from $+3.00 \mathrm{D}$ to $+3.75 \mathrm{D}$ and from $+4.00 \mathrm{D}$ to $+4.75 \mathrm{D}$, respectively constitute $17 \%$ and $7 \%$ of the population and are more present after the age of 55 with the highest prevalence in the age from 65 to 69.

The use of a positive addition in near vision to remedy problems of convergence, accommodation or visual fatigue even in young adults or in the pre-presbyopia phase has been reported in several publications and concerns a part of the population of this study starting from the age of 30 [27] [28] [29] [30].

In many records of this study, it appears that one or more family members use pre-assembled glasses for presbyopia purchased without a prescription in shopping malls or pharmacies and it often happens that these glasses are used after some time also for watching TV and driving. With this inadequate correction there is a higher probability of developing late-onset myopia [31].

The distribution of presbyopia correction in this population is similar to other studies [32] [33]. There are also many people with undercorrected and uncorrected presbyopia and the personal and global impact is reported [34].

Myopia. From the meta-analysis of global data on myopia greater than $0.50 \mathrm{D}$, adults have a mean prevalence of $26.5 \%$ with the highest values in South-East Asia $32.9 \%$ and the lowest in the Americas $16.2 \%$. Seen in smaller populations, these differences are more extreme with a prevalence of $51.0 \%$ in Myanmar and $4.4 \%$ in India [2]. From the Multi-Ethnic Study of Atherosclerosis in the United 
States, it appears that the prevalence of myopia in general was $25.1 \%$, with lower levels $14,2 \%$ in Hispanic participants, $21.5 \%$ in blacks, $31 \%$ in whites, and $37.2 \%$ in Chinese participants [9].

The distribution of the error in the different age groups in this study indicates that more than $80 \%$ of myopic people up to $-4.75 \mathrm{D}$ are 10 to 39 years old. In the myopic population from $-0.25 \mathrm{D}$ to $-0.50 \mathrm{D}, 9 \%$ are $15-19$ years old and the largest group (22\%) is 30 - 34 years old. The population with error $-1.00 \mathrm{D}$ to sph $-3.75 \mathrm{D}$ begins the significant presence of $6 \%$ at $10-14$ years old group and thereafter grows to $17 \%-18 \%$. Both myopia with spherical power greater than $-2.75 \mathrm{D}$ and myopic astigmatism, in general, maintain this same distribution trend in the different age groups with the highest values concentrated between age 25 and 34. Mixed astigmatism and myopia greater than $-4.75 \mathrm{D}$ are most prevalent in the 20 - 34 year-old groups, followed by 35 - 49 year-old groups.

There are very limited data on the population in the Balkans to make statistics or reach regional conclusions. European Eye Epidemiology (E3) Consortium meta-analyzed data from European adult population studies and indicated a prevalence of $23.9 \%$ for astigmatism, $30.6 \%$ for myopia and $25.2 \%$ for hyperopia and one cohort with Greek participants over the age of 60 were included [4]. A recent study reports data on the prevalence of myopia in 1027 children in the Municipality of Prishtina in Kosovo [35]. Two other scientific publications on the region were found: the prevalence of myopia was analyzed from 1738 questionnaires covering the North Greek high school student population and a controlled study involved 200 conscripts in Greek compulsory national service [36] [37].

\subsection{Analysis of Myopic Progression}

This study is similar to the retrospective analysis of eyeglass ordered in optician stores in the Netherlands which investigated the effect of age, stabilization, dioptric power, and myopic progression of 2555 persons of whom $57.3 \%$ were female [13].

The analysis was done on 2751 prescriptions registered from 726 people of whom $20 \%$ had no refractive error changes. A slight increase in myopia from $0.25 \mathrm{D}$ to $0.50 \mathrm{D}$ was found in $42 \%$ of the population while $17 \%$ had myopic progression from $0.75 \mathrm{D}$ to $1.00 \mathrm{D}$. The group of people who had the highest change from $1.25 \mathrm{D}$ to $1.50 \mathrm{D}$, drops to $11 \%$ while the highest myopic progression appears in smaller groups of people.

In this study there is an increase in the number of people who start the first correction of myopia from -0.25 to -0.75 after the age of 20 and undergo a progression of up to $2 \mathrm{D}$ in within 8 - 10 years. The general growth trend and distribution of myopia in age groups of this study resemble with data from the world population reported in international studies [2]. There are projections that 5 billion people will have myopia and 1 billion people will have high myopia in 2050 [38]. 
Only in 2008 and 2009, the group of people with hyperopia was more numerous than the group with myopia. Since 2010, myopic group has prevailed especially in the lower powers from $-0.25 \mathrm{D}$ to $-1.75 \mathrm{D}$. A higher level of school education and a history of eye disease seem to encourage the use of adequate correction and this is also reflected in the database examined in this study [39].

Almost half of the people visited in the Vision Center during the winter and summer holidays live in Europe, the United States and other countries. Lifestyle and different educational pressures lead to diverse myopic progressions in children of a same ethnic group living in different continents [40] [41]. In the conclusions of the guidelines on the management of myopia, the European Society of Ophthalmology in collaboration with the International Myopia Institute indicates outdoor time as the most promising method of intervention followed by medical treatments, contact lenses or glasses [42].

From the analysis of the prescriptions and the descriptions in the accompanying notes, information emerged not only on numerical data but also on collective and personal behaviors of which we were not fully aware in the early stages of the study. The sale of glasses in many optical chains depends on the change of the prescription, leading to different behaviors in cases borderline if the specialist takes a commission. This phenomenon can be present in a slight form also in other countries but it is more evident in contexts where the conflict of interests and consumer protection is not resolved at the legislative level [43].

Looking at the results of prevalence in different age groups and myopic progression helps make more informed decisions about refractive surgery. The concept that, after the growth phase, after the age of 20 - 25, there is no myopic progression is overcome by the reality of the statistical data. A myopic progression is evident up to the age of 30 - 34 even after refractive surgery, with health and economic consequences [44].

There are beliefs, also found in this study, that lead some people not to use glasses or not to update the correction thinking this help stop the progression of ametropias, especially myopia, even if the studies show the opposite [45] [46] [47]. Uncorrected or undercorrected refractive error are significant cause of correctable visual impairment but this is often not understood by the people and authorities who set health care strategies [3] [48].

The strength of this longitudinal retrospective analysis is that this study is conducted on a 13-year database systematically registered in specific software that allows analysis with multiple search criteria. This reduces the margin of error in the statistics and allows cross-referencing of different data. The proportion between the two genders is similar to the population using optical correction [13]. The distribution in age groups is very similar to the demographic data of the resident population in Tirana [12]. Since 2016 every year 50\% of people are registered for the first time in our database and their distribution of refractive errors is similar with the group of people who have taken exams before. The calculation of the progression is based on the differences in myopic correction 
between two exams done at different times in this Vision Center, with the same co-management protocol.

Limitation in the comparison of prevalence data from different studies. During the analysis of the prevalence of each refractive error in this study, we found that different methodologies and population selection criteria in international studies lead to different statistical results. Different cut points are applied to establish the prevalence of hyperopia and the threshold of $+2.00 \mathrm{D}$ is more common in cycloplegic refraction in children while in adults error is considered by the majority of studies if it exceeds +0.50D [2] [14] [18] [23]. In some studies, the cylinder starts from $0.50 \mathrm{D}$ while in the majority of epidemiologic studies the power must exceed 0.50D [24] [25] [26].

Despite having this difference in the statistics, the distribution of ametropias and the course of the progression of myopia remains clear. Besides the analysis of the contexts in which they occur and of the risk factors, other assessments are also useful. Many studies report the importance of using big data, telemedicine, the synergy between different vision care professionals and, above all, putting the user at the center of this process through health education and prevention strategies [49] [50] [51]. This allows the provider to offer different services with quality, sustainable cost and long-term impact.

We believe that the techniques and procedures of the examination assume a fundamental role in prevention. It is imperative that health care strategies and reforms include accommodation, convergence and binocular assessment in all examination protocols in Europe as their impact on refractive error and its progression has been studied and highlighted for many years [52]-[62].

\section{Conclusion}

This 13-year retrospective study of digitally archived data of 19,686 people $(39.5 \%$ $\mathrm{M}$ and $60.5 \% \mathrm{~F}$ ) reported in the urban area of Tirana the prevalence of astigmatism $42 \%$ ( $12 \%$ only astigmatism, $30 \%$ associated with hyperopia or myopia), myopia $25 \%$, hyperopia $22 \%$ and $24 \%$ optical correction with positive lenses for medium and close distances. Analysis of myopia progression (mean spherical equivalent) in adults aged 20 to 39 with refractive errors from $-1.00 \mathrm{D}$ to $-4.75 \mathrm{D}$, indicated that $20 \%$ of people did not experience any changes, $42 \%$ had an increase in myopic correction from $0.25 \mathrm{D}$ to $0.50 \mathrm{D}, 17 \%$ from $0.75 \mathrm{D}$ to $1.00 \mathrm{D}$, $11 \%$ from $1.25 \mathrm{D}$ to $1.50 \mathrm{D}, 5 \% 1.75 \mathrm{D}$ to $2.00 \mathrm{D}$ and $5 \%$ of the population had a myopic progression greater than $2.00 \mathrm{D}$.

\section{Conflicts of Interest}

The author declares no conflicts of interest regarding the publication of this paper.

\section{References}

[1] Freeman, E.E., Roy-Gagnon, M.H., Samson, E., Haddad, S., Aubin, M.J., Vela, C. and Zunzunegui, M.V. (2013) The Global Burden of Visual Difficulty in Low, Middle, and High Income Countries. PLoS ONE, 8, e63315. 
https://doi.org/10.1371/journal.pone.0063315

[2] Hashemi, H., Fotouhi, A., Yekta, A., Pakzad, R., Ostdimoghaddam, H. and Khabazkhoob, M. (2017) Global and Regional Estimates of Prevalence of Refractive Errors: Systematic Review and Meta-Analysis. Journal of Current Ophthalmology, 30, 3-22. https://doi.org/10.1016/j.joco.2017.08.009

[3] Resnikoff, S., Pascolini, D., Mariotti, S.P. and Pokharel, G.P. (2008) Global Magnitude of Visual Impairment Caused by Uncorrected Refractive Errors in 2004. Bulletin of the World Health Organization, 86, 63-70. https://doi.org/10.2471/BLT.07.041210

[4] Williams, K.M., Verhoeven, V.J., Cumberland, P., Bertelsen, G., Wolfram, C., Buitendijk, G.H., Hofman, A., van Duijn, C.M., Vingerling, J.R., Kuijpers, R.W., Höhn, R., Mirshahi, A., Khawaja, A.P., Luben, R.N., Erke, M.G., von Hanno, T., Mahroo, O., Hogg, R., Gieger, C., Cougnard-Grégoire, A., Hammond, C.J., et al. (2015) Prevalence of Refractive Error in Europe: The European Eye Epidemiology (E(3)) Consortium. European Journal of Epidemiology, 30, 305-315.

https://doi.org/10.1007/s10654-015-0010-0

[5] Chiang, S.Y., Weng, T.H., Lin, C.M. and Lin, S.M. (2020) Ethnic Disparity in Prevalence and Associated Risk Factors of Myopia in Adolescents. Journal of the Formosan Medical Association, 119, 134-143. https://doi.org/10.1016/j.jfma.2019.03.004

[6] Gong, Y., Zhang, X., Tian, D., Wang, D. and Xiao, G. (2014) Parental Myopia, near Work, Hours of Sleep and Myopia in Chinese Children. Health, 6, 64-70. https://doi.org/10.4236/health.2014.61010

[7] Hysi, P.G., Choquet, H., Khawaja, A.P., Wojciechowski, R., Tedja, M.S., Yin, J., Simcoe, M.J., Patasova, K., Mahroo, O.A., Thai, K.K., Cumberland, P.M., Melles, R.B., Verhoeven, V., Vitart, V., Segre, A., Stone, R.A., Wareham, N., Hewitt, A.W., Mackey, D.A., Klaver, C., Hammond, C.J., et al. (2020) Meta-Analysis of 542,934 Subjects of European Ancestry Identifies New Genes and Mechanisms Predisposing to Refractive Error and Myopia. Nature Genetics, 52, 401-407.

https://doi.org/10.1038/s41588-020-0599-0

[8] Lai, L., Hsu, W., Kuo, C., Hong, R. and Chen, M. (2014) The Relationship between Myopia and Ocular Alignment among Rural Adolescents. Open Journal of Preventive Medicine, 4, 834-843. https://doi.org/10.4236/ojpm.2014.411094

[9] Pan, C.W., Klein, B.E., Cotch, M.F., Shrager, S., Klein, R., Folsom, A., Kronmal, R., Shea, S.J., Burke, G.L., Saw, S.M. and Wong, T.Y. (2013) Racial Variations in the Prevalence of Refractive Errors in the United States: The Multi-Ethnic Study of Atherosclerosis. American Journal of Ophthalmology, 155, 1129-1138.e1. https://doi.org/10.1016/j.ajo.2013.01.009

[10] Qiu, M., Wang, S.Y., Singh, K. and Lin, S.C. (2014) Racial Disparities in Uncorrected and Undercorrected Refractive Error in the United States. Investigative Oph thalmology \& Visual Science, 55, 6996-7005. https://doi.org/10.1167/iovs.13-12662

[11] Wu, Y., Yi, H., Liu, W., Jia, H., Eshita, Y., Wang, S., Qin, P. and Sun, J. (2012) Risk Factors for Myopia in Inner Mongolia Medical Students in China. Open Journal of Epidemiology, 2, 83-89. https://doi.org/10.4236/ojepi.2012.24013

[12] Bashkia Tiranë (2020) Open Data: Popullsia e Tiranës. https://opendata.tirana.al/?q=popullsia-e-tiran\%C3\%ABs-202

[13] Polling, J.R., Klaver, C. and Tideman, J. (2021) Myopia Progression from Wearing First Glasses to Adult Age: The DREAM Study. British Journal of Ophthalmology. https://doi.org/10.1136/bjophthalmol-2020-316234 
[14] Vitale, S., Ellwein, L., Cotch, M.F., Ferris, F.L. and Sperduto, R. (2008) Prevalence of Refractive Error in the United States, 1999-2004. Archives of Ophthalmology (Chicago, Ill: 1960), 126, 1111-1119. https://doi.org/10.1001/archopht.126.8.1111

[15] Alharbi, A. and Al Harbi, H.D. (2020) An Update of the Recent Developments on Amblypia. Open Journal of Ophthalmology, 10, 241-254.

https://doi.org/10.4236/ojoph.2020.103026

[16] Istek, Ş. (2017) Anisometropia Magnitude and Amblyopia Depth in Previously Untreated Unilateral Amblyopia Patients. Open Access Library Journal, 4, e3565. https://doi.org/10.4236/oalib.1103565

[17] Khalaj, M., Zeidi, I., Gasemi, M. and Keshtkar, A. (2011) The Effect of Amblyopia on Educational Activities of Students Aged 9-15. Journal of Biomedical Science and Engineering, 4, 516-521. https://doi.org/10.4236/jbise.2011.47066

[18] Kempen, J.H., Mitchell, P., Lee, K.E., Tielsch, J.M., Broman, A.T., Taylor, H.R., Ikram, M.K., Congdon, N.G., O’Colmain, B.J. and Eye Diseases Prevalence Research Group (2004) The Prevalence of Refractive Errors among Adults in the United States, Western Europe, and Australia. Archives of Ophthalmology (Chicago, Ill:: 1960), 122, 495-505. https://doi.org/10.1001/archopht.122.4.495

[19] Kulp, M.T., Ciner, E., Maguire, M., Pistilli, M., Candy, T.R., Ying, G.S., Quinn, G., Cy-ert, L., Moore, B. and Vision in Preschoolers-Hyperopia in Preschoolers Study Group (2017) Attention and Visual Motor Integration in Young Children with Uncorrected Hyperopia. Optometry and Vision Science: Official Publication of the American Academy of Optometry, 94, 965-970.

https://doi.org/10.1097/OPX.0000000000001123

[20] Moon, B.Y., Cho, H.G., Yu, D.S. and Kim, S.Y. (2019) Uncorrected Low Hyperopia in Young Subjects Induces Postural Instability Even in Those with Clear Visual Acuity. PLoS ONE, 14, e0224031. https://doi.org/10.1371/journal.pone.0224031

[21] Park, S.H. (2019) Current Management of Childhood Amblyopia. Korean Journal of Ophthalmology: KJO, 33, 557-568. https://doi.org/10.3341/kjo.2019.0061

[22] VIP-HIP Study Group, Kulp, M.T., Ciner, E., Maguire, M., Moore, B., Pentimonti, J., Pis-tilli, M., Cyert, L., Candy, T.R., Quinn, G. and Ying, G.S. (2016) Uncorrected Hyperopia and Preschool Early Literacy: Results of the Vision in PreschoolersHyperopia in Pre-Schoolers (VIP-HIP) Study. Ophthalmology, 123, 681-689. https://doi.org/10.1016/j.ophtha.2015.11.023

[23] Wallace, D.K., Repka, M.X., Lee, K.A., Melia, M., Christiansen, S.P., Morse, C.L., Sprunger, D.T. and American Academy of Pediatric Ophthalmology/Strabismus Preferred Practice Pattern Pediatric Ophthalmology Panel (2018) Amblyopia Preferred Practice Pattern ${ }^{\oplus}$. Ophthalmology, 125, P105-P142.

https://doi.org/10.1016/j.ophtha.2017.10.008

[24] Harvey, E.M., Miller, J.M., Apple, H.P., Parashar, P., Twelker, J.D., Crescioni, M., Davis, A.L., Leonard-Green, T.K., Campus, I. and Sherrill, D.L. (2014) Accommodation in Astigmatic Children during Visual Task Performance. Investigative Ophthalmology \& Visual Science, 55, 5420-5430. https://doi.org/10.1167/iovs.14-14400

[25] Wang, L.L., Wang, W., Han, X.T. and He, M.G. (2018) Influence of Severity and Types of Astigmatism on Visual Acuity in School-Aged Children in Southern China. International Journal of Ophthalmology, 11, 1377-1383.

[26] Huang, L., Yang, G.Y., Schmid, K.L., Chen, J.Y., Li, C.G., He, G.H., Ruan, Z.L. and Chen, W.Q. (2020) Screen Exposure during Early Life and the Increased Risk of Astigmatism among Preschool Children: Findings from Longhua Child Cohort Study. International Journal of Environmental Research and Public Health, 17, 2216. 
https://doi.org/10.3390/ijerph17072216

[27] Han, X.T., Lee, P.-Y., Liu, C. and He, M.G. (2018) Distribution and Progression of Add Power among People in Need of Near Correction. Clinical \& Experimental Ophthalmology, 46, 882-887. https://doi.org/10.1111/ceo.13301

[28] Heus, P., Verbeek, J.H. and Tikka, C. (2018) Optical Correction of Refractive Error for Pre-Venting and Treating Eye Symptoms in Computer Users. The Cochrane Database of Systematic Reviews, 4, CD009877. https://doi.org/10.1002/14651858.CD009877.pub2

[29] O’Leary, C.I., Evans, B. and Edgar, D. (2014) The Effect of Low Refractive Corrections on Rate of Reading. Optometry in Practice, 15, 87-100.

[30] Yammouni, R. and Evans, B.J. (2020) An Investigation of Low Power Convex Lenses (Adds) for Eyestrain in the Digital Age (CLEDA). Journal of Optometry, 13, 198209. https://doi.org/10.1016/j.optom.2019.12.006

[31] Rahman, A., Rahman, S., Faridi, J., Toma, F.T., Huq, A.F. and Khondokar, A.I. (2021) Induced Myopia among Readymade Garment (RGM) Workers. Open Journal of Ophthalmology, 11, 91-98. https://doi.org/10.4236/ojoph.2021.112007

[32] Hickenbotham, A., Roorda, A., Steinmaus, C. and Glasser, A. (2012) Meta-Analysis of Sex Differences in Presbyopia. Investigative Ophthalmology \& Visual Science, 53, 3215-3220. https://doi.org/10.1167/iovs.12-9791

[33] Laughton, D.S., Sheppard, A.L. and Davies, L.N. (2018) Refraction during Incipient Presbyopia: The Aston Longitudinal Assessment of Presbyopia (ALAP) Study. Journal of Optometry, 11, 49-56. https://doi.org/10.1016/j.optom.2017.02.001

[34] Berdahl, J., Bala, C., Dhariwal, M., Lemp-Hull, J., Thakker, D. and Jawla, S. (2020) Patient and Economic Burden of Presbyopia: A Systematic Literature Review. Clinical Ophthalmology (Auckland, N.Z.), 14, 3439-3450. https://doi.org/10.2147/OPTH.S269597

[35] Flaka, S., Mire, H., Fitore, S., Fjolla, S. and Avdyl, S. (2020) Prevalence of Myopia in Pre-School and School Children in the Municipality of Prishtina in Kosovo. Open Journal of Ophthalmology, 10, 33-43. https://doi.org/10.4236/ojoph.2020.101005

[36] Konstantopoulos, A., Yadegarfar, G. and Elgohary, M. (2008) Near Work, Education, Family History, and Myopia in Greek Conscripts. Eye (London, England), 22, 542-546. https://doi.org/10.1038/sj.eye.6702693

[37] Mavrakanas, T.A., Mandalos, A., Peios, D., Golias, V., Megalou, K., Gregoriadou, A., Delidou, K. and Katsougiannopoulos, B. (2000) Prevalence of Myopia in a Sample of Greek Students. Acta Ophthalmologica Scandinavica, 78, 656-659. https://doi.org/10.1034/j.1600-0420.2000.078006656.x

[38] Holden, B.A., Fricke, T.R., Wilson, D.A., Jong, M., Naidoo, K.S., Sankaridurg, P., Wong, T.Y., Naduvilath, T.J. and Resnikoff, S. (2016) Global Prevalence of Myopia and High Myopia and Temporal Trends from 2000 through 2050. Ophthalmology, 123, 1036-1042. https://doi.org/10.1016/j.ophtha.2016.01.006

[39] COMET Group (2013) Myopia Stabilization and Associated Factors among Participants in the Correction of Myopia Evaluation Trial (COMET). Investigative Ophthalmology \& Visual Science, 54, 7871-7884. https://doi.org/10.1167/iovs.13-12403

[40] French, A.N., O’Donoghue, L., Morgan, I.G., Saunders, K.J., Mitchell, P. and Rose, K.A. (2012) Comparison of Refraction and Ocular Biometry in European Caucasian Children Living in Northern Ireland and Sydney, Australia. Investigative Ophthalmology \& Visual Science, 53, 4021-4031. https://doi.org/10.1167/iovs.12-9556

[41] Rose, K.A., Morgan, I.G., Smith, W., Burlutsky, G., Mitchell, P. and Saw, S.M. (2008) Myopia, Lifestyle, and Schooling in Students of Chinese Ethnicity in Singa- 
pore and Sydney. Archives of Ophthalmology (Chicago, Ill:: 1960), 126, 527-530. https://doi.org/10.1001/archopht.126.4.527

[42] Németh, J., Tapasztó, B., Aclimandos, W.A., Kestelyn, P., Jonas, J.B., De Faber, J., Jan-uleviciene, I., Grzybowski, A., Nagy, Z.Z., Pärssinen, O., Guggenheim, J.A., Allen, P.M., Baraas, R.C., Saunders, K.J., Flitcroft, D.I., Gray, L.S., Polling, J.R., Haarman, A.E., Tideman, J., Wolffsohn, J.S., Resnikoff, S., et al. (2021) Update and Guidance on Management of Myopia. European Society of Ophthalmology in Cooperation with International Myopia Institute. European Journal of Ophthalmology, 31, 853-883. https://doi.org/10.1177/1120672121998960

[43] Shneor, E., Evans, B.J., Fine, Y., Shapira, Y., Gantz, L. and Gordon-Shaag, A. (2016) A Survey of the Criteria for Prescribing in Cases of Borderline Refractive Errors. Journal of Optometry, 9, 22-31. https://doi.org/10.1016/j.optom.2015.09.002

[44] Bullimore, M.A., Reuter, K.S., Jones, L.A., Mitchell, G.L., Zoz, J. and Rah, M.J. (2006) The Study of Progression of Adult Nearsightedness (SPAN): Design and Baseline Characteristics. Optometry and Vision Science: Official Publication of the American Academy of Optometry, 83, 594-604. https://doi.org/10.1097/01.opx.0000230274.42843.28

[45] Chung, K., Mohidin, N. and O’Leary, D.J. (2002) Undercorrection of Myopia Enhances Rather than Inhibits Myopia Progression. Vision Research, 42, 2555-2559. https://doi.org/10.1016/S0042-6989(02)00258-4

[46] Sun, Y.Y., Li, S.M., Li, S.Y., Kang, M.T., Liu, L.R., Meng, B., Zhang, F.J., Millodot, M. and Wang, N. (2017) Effect of Uncorrection versus Full Correction on Myopia Progression in 12-Year-Old Children. Graefe's Archive for Clinical and Experimental Ophthalmology= Albrecht von Graefes Archiv fur Klinische und Experimentelle Ophthalmologie, 255, 189-195. https://doi.org/10.1007/s00417-016-3529-1

[47] Vasudevan, B., Esposito, C., Peterson, C., Coronado, C. and Ciuffreda, K.J. (2014) Under-Correction of Human Myopia-Is It Myopigenic? A Retrospective Analysis of Clinical Refraction Data. Journal of Optometry, 7, 147-152.

https://doi.org/10.1016/j.optom.2013.12.007

[48] Weih, L.M., Hassell, J.B. and Keeffe, J. (2002) Assessment of the Impact of Vision Impairment. Investigative Ophthalmology \& Visual Science, 43, 927-935.

[49] Zhao, J.L., et al. (2015) The Impact of Myopia and High Myopia. Report of the Joint World Health Organization-Brien Holden Vision Institute Global Scientific Meeting on Myopia. http://www.who.int/blindness/causes/MyopiaReportforWeb.pdf

[50] Moore, M., Loughman, J., Butler, J.S., Ohlendorf, A., Wahl, S. and Flitcroft, D.I. (2021) Application of Big-Data for Epidemiological Studies of Refractive Error. PLoS ONE, 16, e0250468. https://doi.org/10.1371/journal.pone.0250468

[51] Li, J.O., Liu, H., Ting, D., Jeon, S., Chan, R., Kim, J.E., Sim, D.A., Thomas, P., Lin, H., Chen, Y., Sakomoto, T., Loewenstein, A., Lam, D., Pasquale, L.R., Wong, T.Y., Lam, L.A. and Ting, D. (2021) Digital Technology, Tele-Medicine and Artificial Intelligence in Ophthalmology: A Global Perspective. Progress in Retinal and Eye Research, 82, Article ID: 100900. https://doi.org/10.1016/j.preteyeres.2020.100900

[52] Allen, P.M. and O'Leary, D.J. (2006) Accommodation Functions: Co-Dependency and Relationship to Refractive Error. Vision Research, 46, 491-505. https://doi.org/10.1016/j.visres.2005.05.007

[53] Azen, S.P., Varma, R., Preston-Martin, S., Ying-Lai, M., Globe, D. and Hahn, S. (2002) Binocular Visual Acuity Summation and Inhibition in an Ocular Epidemiological Study: The Los Angeles Latino Eye Study. Investigative Ophthalmology \& Visual Science, 43, 1742-1748. 
[54] Baker, D.H., Lygo, F.A., Meese, T.S. and Georgeson, M.A. (2018) Binocular Summation Revisited: Beyond $\sqrt{2}$. Psychological Bulletin, 144, 1186-1199. https://doi.org/10.1037/bul0000163

[55] Flitcroft, D.I. (1998) A Model of the Contribution of Oculomotor and Optical Factors to Emmetropization and Myopia. Vision Research, 38, 2869-2879. https://doi.org/10.1016/S0042-6989(98)00087-X

[56] Jiang, B.C. (1995) Parameters of Accommodative and Vergence Systems and the Development of Late-Onset Myopia. Investigative Ophthalmology \& Visual Science, 36, $1737-1742$.

[57] Olsen, T., Arnarsson, A., Sasaki, H., Sasaki, K. and Jonasson, F. (2007) On the Ocular Refractive Components: The Reykjavik Eye Study. Acta Ophthalmologica Scandinavica, 85, 361-366. https://doi.org/10.1111/j.1600-0420.2006.00847.x

[58] Owens, D.A. and Wolf-Kelly, K. (1987) Near Work, Visual Fatigue, and Variations of Oculomotor Tonus. Investigative Ophthalmology \& Visual Science, 28, 743-749.

[59] Rubin, G.S., Muñoz, B., Bandeen-Roche, K. and West, S.K. (2000) Monocular versus Bin-Ocular Visual Acuity as Measures of Vision Impairment and Predictors of Visual Disability. Investigative Ophthalmology \& Visual Science, 41, 3327-3334.

[60] Vera-Diaz, F.A., Bex, P.J., Ferreira, A. and Kosovicheva, A. (2018) Binocular Temporal Visual Processing in Myopia. Journal of Vision, 18, 17. https://doi.org/10.1167/18.11.17

[61] Jiang, B.C., Schatz, S. and Seger, K. (2005) Myopic Progression and Dark Focus Variation in Optometric Students during the First Academic Year. Clinical \& Experimental Optometry, 88, 153-159.

https://doi.org/10.1111/j.1444-0938.2005.tb06688.x

[62] Gwiazda, J.E., Hyman, L., Norton, T.T., Hussein, M.E., Marsh-Tootle, W., Manny, R., Wang, Y., Everett, D. and COMET Group (2004) Accommodation and Related Risk Factors Associated with Myopia Progression and Their Interaction with Treatment in COMET Children. Investigative Ophthalmology \& Visual Science, 45, 2143 2151. https://doi.org/10.1167/iovs.03-1306 